\title{
La coordination dans le discours scientifique en astronomie : problèmes de traduction
}

\section{Marie-Françoise Tassard}

\section{(2) OpenEdition}

\section{Journals}

Édition électronique

URL : http://journals.openedition.org/asp/3477

DOI : 10.4000/asp.3477

ISSN : 2108-6354

\section{Éditeur}

Groupe d'étude et de recherche en anglais de spécialité

\section{Édition imprimée}

Date de publication : 1 décembre 1996

Pagination : 161-179

ISSN : 1246-8185

\section{Référence électronique}

Marie-Françoise Tassard, «La coordination dans le discours scientifique en astronomie : problèmes de traduction », ASp [En ligne], 11-14 | 1996, mis en ligne le 06 mai 2013, consulté le 10 décembre 2020. URL : http://journals.openedition.org/asp/3477 ; DOI : https://doi.org/10.4000/asp.3477

Ce document a été généré automatiquement le 10 décembre 2020.

Tous droits réservés 


\title{
La coordination dans le discours
}

\section{scientifique en astronomie : problèmes de traduction}

\author{
Marie-Françoise Tassard
}

1 Grâce aux progrès des technologies modernes, le regard de l'homme peut désormais atteindre les confins de l'univers, et l'astronomie connaît un regain d'intérêt auprès du grand public. Science la plus ancienne et la plus moderne à la fois, située au carrefour de plusieurs autres sciences (physique, géologie, chimie, etc.), l'astronomie est actuellement hautement médiatisée et voit le volume de ses publications s'accroître chaque jour. C'est sur un corpus de textes de vulgarisation en astronomie que nous avons travaillé.

2 Étant donné l'expansion et l'accélération des échanges internationaux, un nombre croissant de publications paraissent dans le monde. Toutefois, peu de textes de vulgarisation se présentent comme des traductions. Seule, la revue Pour la Science publie la traduction en français des articles parus dans Scientific American. Ces articles sont rédigés par les auteurs eux-mêmes, qui, désireux de se faire connaître au-delà d'une communauté de pairs, s'adressent à un public plus large d'étudiants ou de confrères non spécialistes du domaine. Cette revue s'attache à garder un niveau de scientificité plus élevé que les formes de vulgarisation plus populaires, telles que celles qu'on trouve dans Time ou The Guardian.

3 Nous ne savons encore que peu de choses sur le fonctionnement de ces phénomènes dans le discours scientifique vulgarisé, lequel possède ses propres stratégies et ses propres normes, constituant un genre à part entière parmi les langues de spécialité (LSP) et non une simplification du discours de spécialité.

4 Rappelons que la LSP a pour fonction essentielle d'informer et que la communication repose sur un double système de référence : d'une part le savoir commun de la culture collective courante (world knowledge), d'autre part les connaissances scientifiques propres au domaine, partagées par la communauté ou savoir partagé (shared knowledge). Néanmoins, la frontière entre savoir usuel et savoir scientifique se déplace suivant le 
public et suivant la représentation que se fait l'énonciateur de son public. Dans le discours vulgarisateur, tout comme en traduction, l'adaptation aux connaissances de l'autre est fondamentale. En effet, la vulgarisation (VS) fait passer le lecteur d'un système cognitif à un autre, et la traduction d'un système linguistique et culturel à un autre. De plus, si les savoirs scientifiques ont un caractère transculturel, ce n'est pas le cas des savoirs communs qui varient suivant les cultures.

Il est aujourd'hui admis que la langue de spécialité ne se réduit pas à de la terminologie ; en effet la pensée ne peut exister sans mise en rapport logique des énoncés. Ce sont essentiellement les phénomènes de connexion qui permettent de raisonner. Parmi ceuxci, c'est à la coordination qui assure la liaison de mots, de phrases et d'unités plus larges du texte que nous nous intéressons. Cette dernière assure la mise en place de liens logicosémantiques, indispensables au raisonnement et à l'argumentation. Or il n'existe pas toujours de correspondance terme à terme de ces marqueurs, et cela engendre parfois un bouleversement de l'agencement syntaxique de la phrase et d'unités plus larges du texte.

On peut émettre l'hypothèse que les stratégies mises en place d'une langue à l'autre diffèrent quelque peu. Est-il possible de dégager des constantes dans les problèmes de traduction au sein d'un discours, d'un genre? Que signifie la tendance d'une langue? D'autres paramètres interviennent-ils ? Peut-on mesurer la part de chaque facteur? C'est à ces questions que nous avons tenté de répondre.

7 La coordination assure en partie les liens logico-sémantiques indispensables à l'organisation argumentative du discours. Les modes d'enchaînement des relations logiques impliquent les articulations de conjonction, disjonction, restriction, opposition, cause, conséquence et but. Les relateurs, par leur fonction jonctive, structurent le texte en mettant en place les liens cohésifs nécessaires à la cohérence de l'argumentation et à l'inférence :

As much meaning is contained in the relationship between clauses as in the clauses themselves. If one recognizes the basic relationships between clauses, one can make many inferences. (Miller 1993 : 93)

8 Occupant une place de choix dans l'énonciation d'opérations logiques primordiales dans l'argumentation scientifique, les relateurs structurent également le discours et la pensée. On distingue trois niveaux de la construction logique : le linguistique, le discursif et le cognitif. Pensée et langage se construisent dans une relation de réciprocité.

9 En anglais la coordination semble encore plus difficile à définir qu'en français en raison de l'existence de nombreux connecteurs adverbiaux. Nous empruntons à M. Halliday le terme conjunction pour désigner la coordination au sens large, en réunissant à la fois les coordonnants proprement dits et les connecteurs interphrastiques. Ces marqueurs relient certes des éléments de la phrase simple, mais assurent également la coordination propositionnelle et interphrastique essentielle à la cohésion macrostructurelle :

It is not the relationship between adjacent sentences which help create the

meaning of the text but that between larger blocks. (Miller $1993: 63$ )

10 À l'intérieur de la coordination, les connecteurs adverbiaux se distinguent par leur mobilité et la possibilité d'être précédés d'un coordonnant. Quirk et al. (1990: 927) font apparaitre un continuum dans l'intégration syntaxique des coordonnants et des subordonnants.

11 La coordination n'a pas le monopole de la fonction jonctive. Il existe trois grands types de mise en relation, la coordination, la subordination et la juxtaposition. On oppose 
coordination et subordination car la première laisse les deux éléments sur le même rang syntaxique, alors que la seconde hiérarchise, c'est-à-dire place la proposition subordonnée sous la domination de la principale, crée une relation de dépendance et implique un classement explicite et univoque.

Il semblerait cependant que la coordination introduise une hiérarchie minimale car la relation n'est pas réversible, les éléments de l'Énoncé ne sont pas indépendants. Inversement, la juxtaposition, ou asyndète, se caractérise par l'absence de marqueur de surface, sans qu'il y ait pour autant absence de lien. Il s'agit du degré zéro de la liaison syntaxique et de l'explicitation du lien logique. La coordination entretient ainsi des rapports étroits avec la ponctuation. Thomas Miller souligne l'importance de cette hiérarchie :

The hierarchy of relations contributes as much to the meaning of the text as the words themselves. (Miller 1993 : 55)

La coordination soude syntaxiquement, permet la réduction et l'ellipse, c'est-à-dire l'économie de la répétition d'un élément dans la seconde proposition, renforçant la cohésion et la solidarité. Elle sert également l'intention énumérative. En outre la conjonction est parfois complétée par un adverbe tel que SO, THEN, ou THUS, qui va expliciter la relation et permettre à l'énonciateur de refléter son point de vue de façon plus nuancée.

\section{Une option discursive}

14 Comme pour les autres formes de l'énoncé, le choix d'un type de marqueur est un choix motivé visant à en exploiter les possibilités. Il s'agit d'un choix de parole. Il faut donc qu'il y ait intérêt à avoir recours à la coordination plutôt qu'à un autre type de marqueur. Le plus important est le statut énonciatif que donne le connecteur aux deux énoncés qu'il relie :

On pourrait croire qu'avec ce type d'opérateur on quitte le domaine de l'énoncé (phrase) complexe pour passer dans celui du discours dont il représente les articulations logico-sémantiques (la cohésion discursive)... C'est à un jeu modal très diversifié que l'on a affaire. (Adamczewski 1982 : 343)

15 Le connecteur peut être considéré comme le modal de la phrase complexe puisque par son choix, l'énonciateur montre la manière dont il se prononce sur le rapport logique entre les énoncés et les prend plus ou moins en charge. Les connecteurs transphrastiques ont un statut linguistique légèrement différent mais sont également les outils de la cohésion discursive (Lapaire \& Rotgé 1993 : 191).

16 Parmi les quatre types de liens cohésifs que distinguent Halliday et Hasan (1976), la coordination propositionnelle et interphrastique (conjunction) diffère quelque peu des autres. Du point de vue de la cohésion, c'est l'étude de la fonction qui prime sur l'étude des marques structurelles. Ces liens peuvent être exprimés soit par des conjonctions de coordination, soit par des marqueurs de nature différente: adverbes, locutions adverbiales ou prépositionnelles (discourse adjuncts), soit par absence de marqueur: asyndète.

17 Halliday \& Hasan (id.) distinguent encore quatre relations remplissant chacune une fonction sémantique: la relation additive, adversative, la relation de causalité et la relation temporelle. Le tableau (voir annexe 1) met en évidence la polysémie de certains 
marqueurs car il n'y a pas toujours correspondance entre le signe de surface et les liens de cohérence : ainsi AND relateur d'ordinaire additif peut marquer la cause, la concession ou un lien temporel. On peut penser que le contexte, le système de référence des utilisateurs porte le sens et permet l'interprétation de la relation sémantique qui sera ou non marquée en surface par des connecteurs. L'énonciateur, par le choix des marqueurs de surface, peut influer sur la reconstruction logique du discours par le co-énonciateur. D'autre part, s'il n'y a pas toujours, dans les textes traduits, correspondance terme à terme de ces marqueurs, comment opère le traducteur-énonciateur?

\section{L'opération de traduction}

Reformulation et énonciation seconde d'un discours primaire source, la traduction comporte une phase de décodage et d'encodage. Au cours des opérations de transcodage, le traducteur a déjà reconstruit l'enchaînement logique des concepts et procède également à des séries de choix en fonction de paramètres socio-culturels : le genre, sa propre évaluation du public ciblé, la politique éditoriale et ses propres idiosyncrasies. Lors des opérations de transcodage

... c'est le non-dit, l'implicite, qui résiste à la transparence de la traduction parce qu'il n'est pas universel et [qu'il] n'est pas une composante de la seule compétence linguistique. (Fernandez 1987)

19 Acte social de communication et de médiation entre deux cultures, la traduction met en relation deux - ou plusieurs - cultures différentes. Elle passe ainsi d'un système de représentation à un autre :

20 Translation is the exploration of an unbridgeable gap and of a tension between cultures. (Martin \& Hewson $1991: 25$ ).

21 La traduction permet un brassage d'informations, en mettant en contact une multitude de faits culturels, et le traducteur, chargé d'opérer ce transfert, devient alors un véritable médiateur interculturel. En ce qui concerne les publications scientifiques, le commanditaire joue un rôle important en créant le besoin et la commande : c'est avec lui que les auteurs doivent négocier leur texte, en fonction des politiques éditoriales des revues par exemple. L'auteur du texte source, hormis sa dépendance du commanditaire, écrit dans la culture de la langue source pour un lecteur appartenant à la même culture. Une équivalence dans le texte cible ne peut par conséquent qu'être approximative. Enfin, le traducteur n'est ni un être transparent, ni un être culturellement neutre. Plusieurs paramètres de nature individuelle peuvent influer sur ses choix langagiers. Or c'est par lui que passe la communication. Devant interpréter le texte, il risque de le faire d'une manière univoque, réduisant les choix possibles et imposant les siens. Hatim et Mason (1990) distinguent trois grandes étapes dans l'opération traduisante :

23 a) le traducteur est lecteur du texte source. Il devra le comprendre, avoir accès aux connaissances spécifiques explicites et implicites, saisir les intentions discursives.

b) en tant que médiateur : il opère le transfert du sens, assure le relais entre deux langues (grammaire et lexique), entre deux cultures, deux rhétoriques.

c) en tant qu'auteur du texte cible, il évalue la qualité, la lisibilité, décide si ce texte est conforme au type de discours, aux conventions de la communauté et du genre, 
26 Le traducteur pose une véritable "équation culturelle» afin d'adopter la meilleure stratégie possible, car c'est l'ensemble du discours qu'il faut rendre: "The act of translating includes a cultural equation » (Martin \& Hewson 1991 : 51).

Par la formule « A Variational Approach », ces deux derniers auteurs résument la démarche $\mathrm{du}$ traducteur. Traduire consiste à formuler une série d'équivalents possibles, en procédant à des comparaisons successives jusqu'au choix définitif.

It is on those formulational strategies that the translator works, not on referential

truths. [...] Translation is a comparative and adjustable process. (ibid.)

Comment le traducteur procède-t-il au choix définitif ? Existe-t-il un choix unique et définitif? Il semble que non. Peut-on dégager des constantes dans les problèmes rencontrés par les traducteurs? Un grand nombre de traductions sont publiées dans le monde, des correspondances socio-culturelles existent. Enfin que signifie la notion de fidélité, ou plutôt où commencent la réécriture et l'adaptation dans un genre où le contexte intertextuel, c'est-à-dire l'accumulation de références aux textes antérieurs et au savoir déjà construit est déterminant? Lorsque le chercheur argumente afin de convaincre son lecteur de l'intérêt de son travail, il se place dans le créneau que lui laisse l'état de la recherche dans sa discipline : «The text itself becomes a system of designating the common system and displacing it " (Martin \& Hewson 1990 : 90). Quelle marge de manœuvre reste-t-il pour le traducteur? Dans la pratique, quelle est la part des contraintes génériques et normatives?

29 C'est la cohérence du texte que la traducteur doit préserver - la cohérence n'est pas de nature linguistique, mais relève d'une intention discursive et reste la même d'une langue à l'autre (Hatim \& Mason 1990: 207). Le traducteur doit établir ses stratégies non seulement en fonction des intentions rhétoriques de l'auteur, mais aussi de l'idée qu'il se fait du savoir de son lecteur. C'est lui qui décide de la part d'implicite ou d'explicite qu'il doit laisser. Remarquons qu'il

arrive souvent que la traduction se caractérise par des effets d'explicitation, c'est-àdire à un plus grand degré d'implicite dans le TS correspond un plus grand degré d'explicite dans le TC. (Fernandez 1987)

30 C'est également en fonction de sa propre ignorance que le traducteur va déduire celle de son lecteur, et donc prendre en charge la reconstruction logique une fois son travail de recherche documentaire accompli. Or, le travail de recherche en langue de spécialité ne concerne pas seulement la compréhension de la terminologie : les véritables problèmes sont d'ordre notionnel. Il s'agit de comprendre non seulement les concepts mais aussi les relations entre les concepts (Lavault 1994 : 45).

\section{Analyse du corpus}

Notre enquête porte sur un corpus de huit articles récents, publiés dans Scientific American et leurs traductions dans Pour la Science. Nous avons limité notre champ d'études à une seule discipline afin de ne pas prendre en compte d'éventuelles différences propres au discours de chaque science. En comparant les stratégies mises en place dans le texte source avec celles du texte cible, nous tentons de repérer des phénomènes suffisamment récurrents pour être pertinents. Nos observations se font dans le sens français-anglais. Les articles de Scientific American, ont été traduits par des spécialistes du domaine et non des traducteurs de formation linguistique. Le traducteur s'adressant à des nonspécialistes devient alors médiateur interdisciplinaire. Les publications de Scientific 
American s'adressent soit à un public non spécialiste du domaine, mais possédant une culture scientifique, soit à un public étudiant. Elles diffèrent en cela de la majorité des publications vulgarisatrices plus populaires et reflètent une tension entre deux pôles : un pôle vulgarisateur, qui s'écarte de la recherche et a pour fonction d'informer des nonspécialistes sous une forme plus distrayante et facilement abordable; et un pôle didactique, visant à instruire et à former de futurs spécialistes et se rapprochant de l'article de recherche.

Notre enquête porte sur la relation de causalité, relation privilégiée dans l'argumentation scientifique. Il s'agit, en effet, de textes à dominante argumentative, dont l'objectif, rappelons-le, est de convaincre :

Argumentation naturally incorporates items of information although its main purpose is not to inform but to evaluate matters and to convince the reader. (Tirkkanen Condit 1985)

Soutenir une thèse revient à la défendre contre d'autres thèses.

\section{La relation de causalité}

La relation de causalité relie une origine et un après, une cause et une conséquence selon l'ordre des éléments. On a « $P$ parce que $Q$ » ou « $Q$ donc $P$ ». Cette relation est fréquente dans le texte à dominante argumentative où l' on peut s'attendre à trouver une gamme variée de connecteurs de causalité. Dans le schéma suivant : "Situation-ProblemSolution-évaluation " «transition to the Solution component is often marked by such connectives as thus, so, therefore. » (S.Tirkkanen Condit, 1985). Pour Halliday \& Hasan le marqueur représentatif est SO, qui se comprend comme " as a result of this ». En anglais, la conjonction de coordination AND - suivie ou non d'un connecteur adverbial- marque cette relation, ainsi que FOR. En français, deux conjonctions de coordination sont impliquées : CAR et DONC (les grammairiens sont d'ailleurs partagés quant au statut de coordonnant de FOR et de CAR). Quant à DONC il peut avoir la mobilité d'un connecteur adverbial. A l'exception de FOR, la relation cause-conséquence en anglais est assurée par des connecteurs adverbiaux.

Nous examinerons les connecteurs adverbiaux les plus répandus: SO, THUS, THEREFORE, dont nous définissons tout d'abord le sens de base, certains connecteurs indiquant une plus grande rigueur du raisonnement comme HENCE, ainsi que des connecteurs spécialisés tels que THEREBY ou HENCEFORTH que la langue scientifique semble s'Être appropriés (Vidalenc, ASp 1, 1993 : 396).

\section{Sens de base des connecteurs}

En tant que connecteur, SO indique conformité, similitude et sert à exprimer une conséquence. Dans leur ouvrage commun Cohesion in English, Halliday et Hasan rappellent que le mot causalité est un terme qui regroupe quatre notions: le résultat, la conséquence, la raison et le but. Ceci explique que SO, conjonction, puisse être paraphrasé par « as a result of this, for this reason, for this purpose / with the intent that » (Lapaire \& Rotgé, 1991: 286). So lie et sémantise le lien, en conférant à ce dernier le trait de causalité. SO est anaphorique et établit un rapport de congruence entre la cause et l'effet ; il marque une causalité libérée du carcan des procédures logiques. THUS et THEREFORE laissent entrevoir une activité cérébrale intense et méthodique de la part de 
l'énonciateur. THUS a souvent le sens d'une reprise validatrice, son caractère conclusif le rend plus proche de AINSI que de la reprise sèchement autoritaire et factuelle signifiée par DONC. THEREFORE est tourné vers l'avant, vers l'origine et laisse entrevoir un travail mental organisé. Lorsqu'il exprime la conséquence, il peut être résultatif et être tourné vers l'après. Il correspond à la démarche canonique : prémisse puis conclusion ou encore introduction d'une cause et introduction de sa conséquence (ibid. : 290).

DONC, quant à lui, peut avoir la mobilité d'un connecteur adverbial. Il peut marquer une simple reprise conclusionnelle (notamment après une digression), ou une opération d'inférence (Culioli 1990).

\section{Une stratégie de la ponctuation}

Le tableau 1 en annexe 2 montre la récurrence des traductions de AND modulé par un connecteur adverbial. Le tableau 2 en annexe 2 montre les traductions de ces connecteurs seuls. En comparant les tableaux 1 et 2, on peut constater que la présence de AND semble supprimer le choix de la dissociation comme équivalent, les traducteurs ont conservé la soudure marquée par le coordonnant. D'autre part, les traducteurs font peu de différence sur le plan sémantique entre SO, THUS et THEREFORE, précédé ou non de AND : c'est la fonction qui prime.

Le trait le plus frappant ensuite est la récurrence de la traduction par deux points. Or il convient de distinguer les deux points de la dissociation (traduction 0 ou lien minimum). L'emploi des deux-points correspond à une stratégie de la ponctuation. En effet,

... s'il n'y a pas subordination, il n'y a pas non plus dissociation, ceci d'autant plus que l'interprétation sémantique peut s'apparenter à celle d'une subordonnée de type : « Prop1 BECAUSE Prop2. » (Lapaire \& Rotgé 1991 : 554)

En fait, les deux points fournissent une relation causale suffisamment vague: justification, expansion, réponse à une attente. Ils annoncent, tendent le texte vers l'après, alors que le texte anglais marque plus fortement la reprise anaphorique suivie d'une conséquence. Les traducteurs, en l'absence d'équivalent exact en français (Vidalenc 1993 : 396), ont recours à deux points. Ainsi le texte français est moins lié, mais joue sur l'effet de proximité. Dissociation et deux-points simplifient la lecture et en accélèrent le rythme, maintenant ainsi l'intérêt du lecteur. La vulgarisation ne doit pas ennuyer.

Cependant une étude plus précise montre que ces traductions sont des choix de parole, motivés, et ne se limitent pas à des équivalents linguistiques. Ainsi, l'on constate qu'avec une fréquence égale, les mêmes connecteurs sont traduits par deux points dans certains cas, et par une subordination dans d'autres (tableaux 1 et 2).

41 Dans les exemples 1 et 2, il s'agit du pôle vulgarisateur du discours apportant une culture scientifique : récit des déboires du chercheur, histoire des découvertes par les autres chercheurs. Dans l'exemple 3, il s'agit d'informations scientifiques considérées par le traducteur comme directement accessibles pour lecteur. Les traducteurs ont choisi les deux points.

Ex. 1 So we settled, rather arbitrarily, on 21 actuators.... arbitrairement nous avons choisi d'utiliser 21 vérins.

Ex. 2 Nasa chose our proposal, and so began the years of toil...Notre projet fut retenu par la NASA : les années de dur labeur commencèrent 
Ex. 3 A white dwarf packs a mass roughly equal to the sun's, within a volume equal to the earth's, and therefore produces tremendous gravitational forces.Une naine blanche concentre une masse équivalente à celle du soleil dans un volume égal à celui de la Terre : les forces gravitationnelles sont 100 fois supérieures...

$42 \mathrm{Au}$ contraire, dans les exemples suivants, il s'agit de l'argumentation scientifique véhiculant un savoir plus complexe ou bien l'élément nouveau argumenté par l'auteur de l'article. Il y a nécessité de convaincre. En subordonnant, les traducteurs commentent, hiérarchisent les énoncés, et explicitent les liens logiques. Par cette prise en charge, ils sélectionnent l'élément important à leurs yeux.

Ex. 4 Venus is more than 20 times that old, so some mechanism must replenish the water.Comme Venus est plus de 20 fois plus vieille que cela, on suppose que...

Ex. 5 galaxies will be much darker and [...] so there will be fewer planets capable of supporting life.les galaxies seront beaucoup plus sombres et [...] de sorte que les hospitalières seront bien moins nombreuses.

D'autre part, nous avons remarqué précédemment que AND pouvait avoir une coloration consécutive. Dans l'exemple suivant, apparaissent la prise de position du traducteur par rapport à l'énoncé, et peut-être sa volonté, en tant que spécialiste et pédagogue, de faciliter la lecture, ici de signaler que dans une démarche scientifique, rien n'est définitivement acquis.

Ex. 6 Over the years, astronomers had studied it extensively and felt that...Elle avait tant été étudiée que les astronomes pensaient connaître....

\section{Coordination et rigueur scientifique} AINSI, AUSSI, et DONC. Les nuances sémantiques entre SO, THUS, THEREFORE et HENCE ne sont pas sensibles dans notre tableau. La présence de THEREBY et HENCEFORTH reste discrète dans notre corpus. HITHERTO et THEREIN sont absents. Or J. L. Vidalenc écrit :

Le paradoxe de la présence dans des textes de discipline de pointe, de marqueurs considérés comme archaïques dans la langue de tous les jours, peut s'expliquer par la présence de cette racine en TH. HITHERTO, THEREBY, THEREIN, en assurant un lien logique avec ce qui vient d'être écrit contribuent à étayer la rigueur du raisonnement. (1993: 395) présence est-elle proportionnelle au niveau de scientificité du texte? HENCE. Les traducteurs n'emploient pas unanimement DONC comme équivalent de HENCE, et semblent hésiter, devant la rigueur et la sécheresse de ce connecteur en français lorsqu'il marque une opération d'inférence, mais l'emploient dans la majorité des cas pour marquer une reprise conclusionnelle dans une narration (Culioli 1990).

Ex. 1 Hence I engaged four graduate students...J'accueillis donc quatre étudiants...

Ex. 2 So they had to be reconfigured to meet NASA's spaceworthinessNous les avons donc adaptés aux normes requises par la NASA. subordination : il y a prise en charge énonciative de la déduction.

Ex. 3 ...hence...dont on déduit que...

Dans notre corpus seul THEREBY témoigne de la rigueur du discours de spécialiste. L'utilisation des connecteurs de cause correspond au genre et au niveau de scientificité de 
la revue, la rigueur des démonstrations scientifiques restant l'exclusivité du discours de spécialiste. En français, le discours jouerait moins sur la variété des connecteurs que sur le degré d'intégration syntaxique. La subordination pourrait être un phénomène compensatoire. La tendance à y recourir s'accentue avec la rigueur de la démonstration et la prise en charge énonciative. De surcroît, le lecteur est novice, et dépendant du traducteur qui a déjà reconstruit l'enchaînement notionnel et établi ses stratégies textuelles afin de convaincre son lecteur. Bien que les phénomènes que nous venons d'observer se répartissent sur l'ensemble des articles, il semble qu'il y ait des préférences, chez certains, pour la traduction par deux points. L'un des articles étudiés accusait un rythme beaucoup plus rapide. Ces divergences peuvent témoigner d'idiosyncrasies individuelles difficilement mesurables.

\section{Cohésion du mouvement argumentatif}

Observons le tableau 0 :

Tableau 0

\begin{tabular}{|l|l|l|}
\hline TS & schéma logique & liaison syntaxique \\
\hline & Si P est vrai, alors Q & (dissociation) \\
\hline & Si Q est vrai alors R & In that case \\
\hline & Si R est vrai, alors S & therefore \\
\hline & Si S alors T & That would amount to... \\
\hline TC & schéma logique & liaison syntaxique \\
\hline & P alors X (X= Q+R) & ( ( éT ) \\
\hline & Y (Y=S +T) & $;($ éT ) \\
\hline
\end{tabular}

Ex. 1 a leading expert proposed that interstellar matter might be distributed quite unevenly. Its density in many directions might be only one tenth of the average. In that case, extreme ultraviolet radiation would penetrate 10 times farther than normally assumed. Therefore, a volume of space 1,000 times greater than was commonly believed to exist would be observable by UV light. That would amount to a 1,000-fold increase in the potential number of such sources...que la matière stellaire n'était pas répartie uniformément: Dans de nombreuses directions sa densité pouvait n'être égale qu'à un dixième de la valeur moyenne et le rayonnement ultraviolet lointain devait pénétrer dix fois plus loin que prévu; un volume mille fois plus grand était observable et 1000 fois plus de sources devaient être visibles...

Le tableau ci-dessus tente de schématiser le type de modifications observées dans l'exemple cité. Nous essayons de montrer comment ces modifications au niveau des marqueurs de surface peuvent influer sur la lecture ou provenir d'une lecture différée qu'est celle du traducteur. Il s'agit d'énoncés en cascade: l'explication comporte une démonstration en plusieurs temps. En anglais, on constate une progression dans la 
rigueur des soudures syntaxiques anaphoriques à mesure que le raisonnement progresse. Pas à pas, l'énonciateur s'appuie sur l'hypothèse précédente. À chaque élément supplémentaire, le connecteur marque un effort croissant. Les éléments sont pris en compte un à un jusqu'à la reprise conclusive. En français les deux points annoncent une expansion et tendent le texte - et l'attention du lecteur - vers l'après. Il s'agit d'une cataphore permettant au lecteur de savoir ce qu'il va lire. Les éléments de la démonstration sont également soudés deux à deux et semblent déjà classés. Il semble que le traducteur-énonciateur simplifie et uniformise la langue. Les liens logiques sont alors plus implicites en français, la stratégie argumentative est simplifiée. Le traducteur a raisonné avant son lecteur, comme le professeur a appris avant l'étudiant, et le spécialiste avant le non-spécialiste.

\section{FOR et la justification}

Le sens de base de FOR a déjà été posé. L'équivalent de FOR en français est en général CAR. Sur le plan syntaxique les grammairiens sont partagés quant à l'appartenance de FOR à la catégorie des conjonctions de coordination ou de subordination (annexe 3). Sur le plan sémantique, il s'agit d'une justification. Rappelons qu'avec CAR l'énonciateur justifie le dire antérieur et commente les motifs de l'énonciation (Lapaire \& Rotgé 1991: 568). De plus dans son article, Deléchelle écrit que CAR et FOR rhématisent l'énoncé et revendiquent la validité des faits ; ils ont donc une valeur polémique.

Lorsque que l'on relève les équivalents de CAR dans notre corpus, le trait le plus frappant est tout d'abord l'absence de FOR, puis la forte proportion de non-traductions. Sur 22 occurrences de CAR, aucune ne correspond à FOR, 11 ont pour équivalent 0,7 correspondent à BECAUSE. Halliday remarque la rareté de FOR en anglais. Delechelle fait la même observation, bien que son corpus appartienne à un genre différent.

\section{CAR, BECAUSE et la causalité directe}

Citons les exemples suivants:

Ex. 1 A different type of wavefront sensor is generally used with laser beacons because it can handle... Un type différent de détecteur de front d'onde est généralement utilisé avec les balises laser, car il peut...

Ex. 2 These observations are especially valuable because the spot appears only once every 60 years or so.L'observation de cette "grande tache blanche 'est précieuse, car cette tache n'apparaît qu'une fois tous les 60 ans.

Ex. 3 Researchers refer to this wake structure as an induced magnetotail because...Ce sillage semble être une magnéto-queue induite, car il provient du champ magnétique.

Ex. 4 Laser beacons are useless for pointing because they are not fixed in the sky...Les balises laser ne guident pas les télescopes, car elles n'ont pas de position fixe.

Par opposition à FOR, BECAUSE fournit la justification d'un procès. Ce subordonnant peut être paraphrasé par « by that cause » et introduit une causalité directe, à propos de faits en général vérifiables (Lapaire \& Rotgé 1991: 568). BECAUSE établit une rupture entre les deux propositions de la phrase. La subordination sélectionne les éléments. Alors que BECAUSE établit un lien de type causal, il n'en va pas de même pour FOR : «Les énoncés de type causal placent le rapport entre $P$ et $Q$ sur le plan des faits alors que les justificatifs concernent le dire» (Deléchelle 1983). En traduisant BECAUSE par CAR le traducteur- 
énonciateur ne se situe plus sur le plan du factuel mais justifie le dire face au lecteur ignorant ou méfiant.

\section{CAR et le lien 0 en anglais}

Citons les exemples suivants:

Ex. 1 The atmosphere of Venus is far richer than the earth in sulfur- containing gases, primarily sulfur dioxide. On the earth, rain efficiently removes similar sulfur gases from the atmosphere. L'atmosphère de Vénus est beaucoup plus riche en gaz souffrés que celle de la terre, surtout en dioxyde de soufre, car les pluies ne les lessivent pas.

Ex. 2 Their journey in Appollo 11 across $380000 \mathrm{kms}$ of space sent them back billions of years. Armstrong, Aldrin and the 10 astronauts returned with samples that contain a fascinating history of the moon and earth. Leur voyage de 380000 kms à bord d'Appollo 11 les propulsa quelques milliards d'années en arrière, car les échantillons de roches lunaires racontaient...

Ex. 3 Marking the beginning of a new era in optical astronomy. earthbound optical telescopes,...have always been hindered Pour l'astronomie optique, c'était un grand jour, car les observations faites au sol...

Ex. 4 ...suggesting a close kinship. If the moon had formed elsewhere in the solar system, it would have had a .....'est un indice de proche parenté, car si la lune s'était formée ailleurs dans le système solaire, sa composition...

Ces exemples ont en commun la dissociation dans le texte anglais. Dans les exemples 2, 3, et 4 , l'énoncé antérieur est une généralisation énoncée dans la langue commune, justifiée ensuite par des informations d'ordre scientifique. C'est l'énonciateur lui-même qui généralise ou apprécie pour le lecteur. En outre les segments antérieurs appartiennent au système référentiel commun : suggest a close kinship, sent them back billions of years, etc. Le scripteur anglophone en fait deux assertions indépendantes. Le réseau anaphorique serré de l'anglais renverra le lecteur vers l'avant. Il établira le lien sans trop de difficulté car le segment antérieur appartient à langue commune. En revanche, CAR fournit le signe explicite de la justification qui va suivre.

Dans le premier exemple, il s'agit de données scientifiques mais l'on remarque qu'il y a davantage d'implicite dans le texte traduit. L'anglais énonce la référence à la pluie sur terre, en français cela fait partie de la référence implicite.

Dans les deux cas précédents, le traducteur-énonciateur anticipe une éventuelle réaction de surprise ou de méfiance de la part du destinataire et utilise les valeurs polémiques et dialogiques de CAR afin de mieux convaincre.

Le traducteur scientifique utilise plus souvent la causalité directe lorsqu'il véhicule des données scientifiques factuelles; lorsqu'il s'agit de lier le dire antérieur appartenant à la langue commune, le lien logique entre les deux énoncés n'est pas explicité. Le français doit-il justifier la mise en relation de deux énoncés situés sur deux plans référentiels différents : généralisation dans le savoir commun et faits scientifiques ? La frontière entre savoir scientifique et culture " commune » reste néanmoins assez imprécise, car il s'agit toujours d'un jugement du scripteur sur son lecteur. Il semble qu'en français de traduction, le traducteur dans les deux cas, ait recours à CAR afin de justifier et de revendiquer la validité des faits, commentant ainsi les motifs de l'énonciation. Dans la relation causale, peut-être plus qu'ailleurs, « le connecteur apparaît comme le modal de la phrase » (Adamcewski 1982: 334), car il y a dans le texte scientifique une forte prise en charge de l'argumentation. 
57 CAR a ici une valeur polémique (Deléchelle 1983) et dialogique : dans le dialogue qui s'instaure avec le lecteur, il introduit à la fois une revendication sur la validité du dire et une précaution oratoire, où l'énonciateur anticipe la réaction du destinataire. «A text is interactive. The writer of an argumentative text anticipates challenging questions from the reader [...] and he is prepared to provide evidence on points which are not expected to be based on shared knowledge or values " (Tirkanen Condit 1985). L'objectif de l'argumentation n'est-il pas, avant tout, de convaincre?

\section{Conclusion} stratégies destinées à convaincre diffèrent quelque peu. Nous avons constaté une tendance dans le texte français à regrouper les notions, mais avant d'invoquer une tendance de la langue à privilégier le notionnel sur le factuel, il convient de ne pas perdre de vue le fait que la traduction est une énonciation seconde et différée, issue d'opérations de transcodage au cours desquelles le traducteur a déjà opéré un classement notionnel, ce qui peut le conduire à surdéterminer le texte.

61 Outre l'objectif didactique de ces publications, nous avons observé une apparente uniformisation et simplification des stratégies argumentatives qui peut s'expliquer par la politique éditoriale qui cherche à normaliser et à uniformiser l'argumentation afin de gommer tant que faire se peut les paramètres dépendant trop des représentations individuelles. La fonction prime alors sur la richesse du style. Si la mise en discours du texte source répond à des contraintes d'ordre générique, au public ciblé, au scripteur et à la politique éditoriale, la traduction apparaît comme un discours gigogne (la volonté d'uniformiser pourrait ne pas échapper à cet effet). Elle comporte des phénomènes qui lui sont propres et qui, s'ils sont caractérisables, n'en restent pas moins difficilement mesurables. Nous ne savons encore que peu de choses sur la modélisation des discours et des langues. Avant d'invoquer des tendances de notre langue ou de notre culture, il convient de distinguer les facteurs présidant à la mise en discours et qui font de tout texte une production linguistiquement et socialement déterminée. Nous sommes loin de 
la soi-disant objectivité du texte scientifique. C'est le discours qui transforme les faits et donne sa propre version de la réalité.

\section{BIBLIOGRAPHIE}

Adamczewski, H. 1981. Grammaire linguistique de l'anglais. Paris : Ophrys.

Culioli, A. 1990. Pour une linguistique de l'énonciation. Paris : Ophrys.

Deléchelle, G. 1983. « En effet et ses traductions en anglais ». Tréma 8, 49-67.

Fernandez, M.J. et al. 1987. « Traduction et vulgarisation scientifique ». DISCOSS 3.

Halliday, M. and Hasan. 1976. Cohesion in English. Londres : Longman.

Hatim, B. and I. Mason. 1990. Discourse and the Translator. Londres/New York : Longman.

Guillemin Flescher, J. 1982. Syntaxe comparée du français et de l'anglais. Paris : Ophrys.

Lapaire J.R. et W. Rotgé. 1991. Linguistique et grammaire de l'anglais. Toulouse : éd. du Mirail.

Lavault E. « Quelle culture pour le traducteur spécialisé ? ». ASp 5-6, 45-53.

Martin J. \& L. Hewson. 1991. Redefining Translation: a Variational Approach. New York : Routledge.

Miller, T. 1993. « The introduction to the research article from a discourse perspective ». ASp 2, 55-68.

Tirkkanen Condit, S. 1985. Argumentative Text Structure and Translation. University of Jyväskyläa. Swales, J. 1990. Genre Analysis. Cambridge : Cambridge University Press.

Vidalenc, J.-L. 1993. «Un apport de l'analyse du discours à l'apprentissage de la rédaction d'articles scientifiques ». ASp 1, 383-401.

\section{Liste des articles analysés}

A1) «Early results from the Hubble Space telescope ». Scientific American, June 92. « Les premiers résultats du télescope spatial Hubble ». Pour la science, août 92.

A2) «How the Milky Way Formed » Scientific American,January 93.«Comment s'est formée la Voie lactée ». Pour la science, mars 93.

A3) « The Pioneer mission to Venus ». Scientific American, April 94.« La mission Pioneer sur Vénus ». Pour la science, juin 94.

A4) « Adaptive optics ». Scientific American, August 94.« L'optique adaptative ». Pour la science, octobre 94.

A5) «The scientific legacy of Apollo ». Scientific American, July 94.« L'héritage scientifique des missions vers la lune ». Pour la science, sept. 94

A6) « Extreme ultraviolet astronomy ». Scientific American, August 94.« Le ciel ultraviolet ». Pour la science, octobre 94. 
A7) « The evolution of the universe ». Scientific American, October 94.« L'évolution de l'univers ». Pour la science, décembre 94.

A8). »The birth and death of Nova V1974 Cygni ». Scientific American, January 95.« Vie et mort de la nova V1974 Cygni ». Pour la science, mars 1995.

\section{ANNEXES}

\section{Annexe 1}

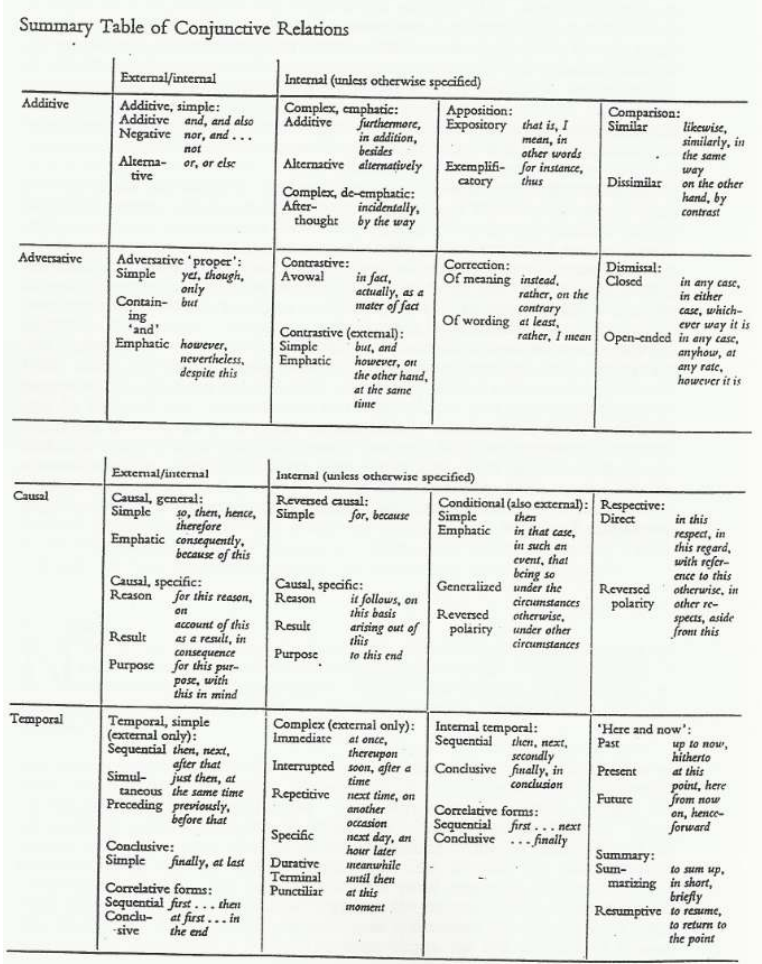

Annexe 2

Tableau 1. Récurrence des traductions de AND + adverbe

\begin{tabular}{|l|l|l|}
\hline TS & TC & fréquence \\
\hline AND SO & $\vdots$ & 2 \\
\hline AND THUS & $\vdots$ & 2 \\
\hline & 0 & 1 \\
\hline & ET DONC & 1 \\
\hline & autres & 2 \\
\hline AND THEREFORE & $:$ & 2 \\
\hline AND HENCE & - & 1 \\
\hline & ET & 1 \\
\hline & subordination & 1 \\
\hline
\end{tabular}

Tableau 2. Traduction des connecteurs adverbiaux 


\begin{tabular}{|l|l|l}
\hline TS & TC & fréquence \\
\hline SO & $:$ & 2 \\
\hline & 0 & 5 \\
\hline & subordination & 5 \\
\hline & AINSI, DONC & 3 \\
\hline THUS & $\vdots$ & 1 \\
\hline & 0 & 4 \\
\hline & ET & 1 \\
\hline & adverbe & 3 \\
\hline THEREFORE & $:$ & 1 \\
\hline & 0 & 2 \\
\hline HENCE & adverbe & 3 \\
\hline THEREBY & DONC, AINSI & 4 \\
\hline & $:$ & 1 \\
\hline & subordination & 2 \\
\hline
\end{tabular}

Annexe 3

Occurrences de CAR et ses équivalents

\begin{tabular}{|l|l|l|}
\hline français & anglais & 22 \\
\hline CAR & 0 & 12 \\
\hline CAR & BECAUSE & 7 \\
\hline CAR & autres & 3 \\
\hline
\end{tabular}

\section{RÉSUMÉS}

L'objet de cette étude contrastive est de vérifier l'hypothèse selon laquelle les stratégies discursives mises en place par la coordination diffèrent quelque peu en français et en anglais de spécialité. L'observation d'un corpus d'articles de vulgarisation scientifique de bon niveau prélevé dans Scientific American et de leurs traductions prélevées dans Pour la science a permis de confirmer cette hypothèse. L'étude de la mise en discours des quatre relations logiques montre que dans le Texte Cible (TC), la recherche devient plus impersonnelle, les éléments ludiques ou anecdotiques sont atténués au profit des concepts et de l'argumentation scientifique. Le traducteur met en place des liens syntactiques plus ou moins explicites (du lien $\mathrm{O}$ à la subordination) lors de la prise en charge des rapports logiques entre les Énoncés selon son appréciation des savoirs partagés. Le spécialiste français s'adresse au non-spécialiste d'une manière plus didactique. Toutefois, avant d'entrevoir des tendances linguistiques et culturelles, il convient d'isoler certains facteurs propres à la traduction, Énonciation seconde, au traducteur, et aux contraintes génériques qui, s'ils sont caractérisables, n'en restent pas moins difficilement mesurables.

Discourse strategies in establishing logical links between clauses and larger units of the text have been assumed to be somehow different in English and French specialised discourses. A contrastive study of the four conjunctive relations shows that the Target Text contains less personal elements, and focuses on concepts and scientific argumentation. According to his own appreciation of the reader's Culture, the translator chooses a more or less explicit syntactic link (from asyndetic coordination to subordination). The French specialist addresses the nonspecialist in a more didactic manner. Rather than linguistic and cultural differences, the present study highlights the importance of other factors: the process of Translation itself, the role of the translator and his evaluation of the reader's previous knowledge, the public targeted and genredetermined constraints which can be characterised, although they remain difficult to measure. 
INDEX

Mots-clés : argumentation, astronomie, causalité, cohésion, coordination, explicite, implicite, savoir partagé, stratégie discursive, traduction, vulgarisation scientifique

Keywords : astronomy, cause-effect relationship, conjunction, discourse strategy, explicit, implicit, popularisation of science, shared knowledge, translation

\section{AUTEUR}

\section{MARIE-FRANÇOISE TASSARD}

Marie-Françoise Tassard est PRCE à la Faculté des sciences de l'Université d'Orléans. mariefrancoise.tassard@univ-orleans.fr 